\title{
Nuclear-mass dependence of azimuthal beam-helicity and beam-charge asymmetries in deeply virtual Compton scattering
}

A. Airapetian, ${ }^{12,15}$ N. Akopov, ${ }^{26}$ Z. Akopov, ${ }^{5}$ M. Amarian, ${ }^{6}$ E. C. Aschenauer,,${ }^{6 *}$ W. Augustyniak, ${ }^{25}$ R. Avakian, ${ }^{26}$ A. Avetissian, ${ }^{26}$ E. Avetisyan, ${ }^{5}$ B. Ball, ${ }^{15} \mathrm{~S}$. Belostotski, ${ }^{18} \mathrm{~N}$. Bianchi, ${ }^{10}$ H. P. Blok, ${ }^{17,24}$ H. Böttcher, ${ }^{6}$ A. Borissov, ${ }^{5}$ J. Bowles, ${ }^{13}$ V. Bryzgalov, ${ }^{19}$ J. Burns, ${ }^{13}$ M. Capiluppi, ${ }^{9}$ G. P. Capitani, ${ }^{10}$ E. Cisbani, ${ }^{21}$ G. Ciullo, ${ }^{9}$ M. Contalbrigo, ${ }^{9}$ P. F. Dalpiaz, ${ }^{9}$ W. Deconinck,${ }^{5,15, \dagger}$ R. De Leo, ${ }^{2}$ L. De Nardo, ${ }^{5,15}$ E. De Sanctis, ${ }^{10}$ M. Diefenthaler, ${ }^{8,14}$ P. Di Nezza, ${ }^{10}$ M. Düren, ${ }^{12}$ M. Ehrenfried, ${ }^{12}$ G. Elbakian, ${ }^{26}$ F. Ellinghaus,,${ }^{4}{ }^{\ddagger}$ R. Fabbri, ${ }^{6}$ A. Fantoni,${ }^{10}$ L. Felawka, ${ }^{22}$ S. Frullani, ${ }^{21}$ D. Gabbert,,${ }^{6,11}$ G. Gapienko, ${ }^{19}$ V. Gapienko, ${ }^{19}$ F. Garibaldi, ${ }^{21}$ G. Gavrilov, ${ }^{5,18,22}$ V. Gharibyan, ${ }^{26}$ F. Giordano, ${ }^{5,9}$ S. Gliske, ${ }^{15}$ H. Guler, ${ }^{6}$ V. Guzey, ${ }^{\S}$ S. Haan, ${ }^{6}$ C. Hadjidakis, ${ }^{10}$ M. Hartig, ${ }^{5, \|}$ D. Hasch,,${ }^{10}$ G. Hill, ${ }^{13}$ A. Hillenbrand, ${ }^{6}$ M. Hoek, ${ }^{13}$ Y. Holler, ${ }^{5}$ I. Hristova, ${ }^{6}$ Y. Imazu, ${ }^{23}$ A. Ivanilov, ${ }^{19}$ H. E. Jackson, ${ }^{1}$ H. S. Jo, ${ }^{11}$ S. Joosten, ${ }^{11,14}$ R. Kaiser, ${ }^{13}$ G. Karyan, ${ }^{26}$ T. Keri, ${ }^{12,13}$ E. Kinney, ${ }^{4}$ A. Kisselev, ${ }^{18}$ V. Korotkov, ${ }^{19}$ V. Kozlov, ${ }^{16}$ P. Kravchenko, ${ }^{18}$ L. Lagamba, ${ }^{2}$ R. Lamb, ${ }^{14}$ L. Lapikás, ${ }^{17}$ I. Lehmann, ${ }^{13}$ P. Lenisa, ${ }^{9}$ A. López Ruiz, ${ }^{11}$ W. Lorenzon, ${ }^{15}$ X.-G. Lu, ${ }^{6}$ X.-R. Lu, ${ }^{23}$ B.-Q. Ma, ${ }^{3}$ D. Mahon, ${ }^{13}$ N. C. R. Makins, ${ }^{14}$

S. I. Manaenkov, ${ }^{18}$ L. Manfré,${ }^{21}$ Y. Mao, ${ }^{3}$ B. Marianski, ${ }^{25}$ A. Martinez de la Ossa, ${ }^{4}$ H. Marukyan, ${ }^{26}$ C. A. Miller, ${ }^{22}$ Y. Miyachi, ${ }^{23}$ A. Movsisyan, ${ }^{26}$ V. Muccifora, ${ }^{10}$ M. Murray, ${ }^{13}$ A. Mussgiller,${ }^{5,8}$ E. Nappi, ${ }^{2}$ Y. Naryshkin, ${ }^{18}$ A. Nass, ${ }^{8}$ M. Negodaev, ${ }^{6}$ W.-D. Nowak, ${ }^{6}$ L. L. Pappalardo, ${ }^{9}$ R. Perez-Benito, ${ }^{12}$ M. Raithel, ${ }^{8}$ P. E. Reimer, ${ }^{1}$ A. R. Reolon, ${ }^{10}$ C. Riedl, ${ }^{6}$ K. Rith, ${ }^{8,9}$ G. Rosner, ${ }^{13}$ A. Rostomyan, ${ }^{5}$ J. Rubin, ${ }^{14}$ D. Ryckbosch, ${ }^{11}$ Y. Salomatin, ${ }^{19}$ A. Schäfer, ${ }^{20}$ G. Schnell, ${ }^{6,11}$ K. P. Schüler, ${ }^{5}$ R. Shanidze, ${ }^{8}$ T.-A. Shibata, ${ }^{23}$ V. Shutov, ${ }^{7}$ M. Stancari, ${ }^{9}$ M. Statera, ${ }^{9}$ E. Steffens, ${ }^{8}$ J. J. M. Steijger, ${ }^{17}$ J. Stewart, ${ }^{6}$

F. Stinzing, ${ }^{8}$ S. Taroian, ${ }^{26}$ A. Terkulov, ${ }^{16}$ A. Trzcinski, ${ }^{25}$ M. Tytgat, ${ }^{11}$ A. Vandenbroucke, ${ }^{11}$ Y. Van Haarlem, ${ }^{11, * *}$

C. Van Hulse, ${ }^{11}$ M. Varanda, ${ }^{5}$ D. Veretennikov, ${ }^{18}$ V. Vikhrov, ${ }^{18}$ I. Vilardi, ${ }^{2}$ S. Wang, ${ }^{3}$ S. Yaschenko, ${ }^{8,6}$ H. Ye, ${ }^{3}$ Z. Ye, ${ }^{5}$ W. Yu, ${ }^{12}$ D. Zeiler, ${ }^{8}$ B. Zihlmann, ${ }^{5}$ and P. Zupranski ${ }^{25}$

(HERMES Collaboration)

${ }^{1}$ Physics Division, Argonne National Laboratory, Argonne, Illinois 60439-4843, USA

${ }^{2}$ Istituto Nazionale di Fisica Nucleare, Sezione di Bari, I-70124 Bari, Italy

${ }^{3}$ School of Physics, Peking University, Beijing 100871, People's Republic of China

${ }^{4}$ Nuclear Physics Laboratory, University of Colorado, Boulder, Colorado 80309-0390, USA

${ }^{5}$ DESY, D-22603 Hamburg, Germany

${ }^{6}$ DESY, D-15738 Zeuthen, Germany

${ }^{7}$ Joint Institute for Nuclear Research, RU-141980 Dubna, Russia

${ }^{8}$ Physikalisches Institut, Universität Erlangen-Nürnberg, D-91058 Erlangen, Germany

${ }^{9}$ Istituto Nazionale di Fisica Nucleare, Sezione di Ferrara, and Dipartimento di Fisica, Università di Ferrara, I-44100 Ferrara, Italy

${ }^{10}$ Istituto Nazionale di Fisica Nucleare, Laboratori Nazionali di Frascati, I-00044 Frascati, Italy

${ }^{11}$ Department of Subatomic and Radiation Physics, University of Gent, B-9000 Gent, Belgium

${ }^{12}$ Physikalisches Institut, Universität Gießen, D-35392 Gießen, Germany

${ }^{13}$ Department of Physics and Astronomy, University of Glasgow, Glasgow G12 8QQ, United Kingdom

${ }^{14}$ Department of Physics, University of Illinois, Urbana, Illinois 61801-3080, USA

${ }^{15}$ Randall Laboratory of Physics, University of Michigan, Ann Arbor, Michigan 48109-1040, USA

${ }^{16}$ Lebedev Physical Institute, RU-117924 Moscow, Russia

${ }^{17}$ National Institute for Subatomic Physics (Nikhef), NL-1009 DB Amsterdam, The Netherlands

${ }^{18}$ Petersburg Nuclear Physics Institute, Gatchina, Leningrad region RU-188300, Russia

${ }^{19}$ Institute for High Energy Physics, Protvino, Moscow region RU-142281, Russia

${ }^{20}$ Institut für Theoretische Physik, Universität Regensburg, D-93040 Regensburg, Germany

${ }^{21}$ Istituto Nazionale di Fisica Nucleare, Sezione Roma 1, Gruppo Sanità and Physics Laboratory,

Istituto Superiore di Sanità, I-00161 Roma, Italy

${ }^{22}$ TRIUMF, Vancouver, British Columbia V6T 2A3, Canada

${ }^{23}$ Department of Physics, Tokyo Institute of Technology, Tokyo 152, Japan

${ }^{24}$ Department of Physics, VU University, NL-1081 HV Amsterdam, The Netherlands

${ }^{25}$ Andrzej Soltan Institute for Nuclear Studies, PL-00-689 Warsaw, Poland

${ }^{26}$ Yerevan Physics Institute, 375036 Yerevan, Armenia

(Received 3 November 2009; published 5 March 2010)

\footnotetext{
The nuclear-mass dependence of azimuthal cross-section asymmetries with respect to charge and longitudinal polarization of the lepton beam is studied for hard exclusive electroproduction of real photons. The observed beam-charge and beam-helicity asymmetries are attributed to the interference between the Bethe-Heitler and the deeply virtual Compton scattering processes. For various nuclei, the asymmetries are extracted for both coherent and incoherent-enriched regions, which involve different (combinations of) generalized parton distributions. For
} 
both regions, the asymmetries are compared to those for a free proton, and no nuclear-mass dependence is found.

DOI: 10.1103/PhysRevC.81.035202

\section{INTRODUCTION}

Lepton scattering experiments constitute an important source of information for understanding nucleon structure in the context of QCD. Until recently, this structure was described by two categories of nonperturbative objects, form factors and parton distribution functions (PDFs), which have been measured in elastic and deep-inelastic scattering (DIS) experiments, respectively. In the last decade, generalized parton distributions (GPDs) [1-4] have been recognized as a key concept for the description of hard exclusive processes. GPDs offer a multidimensional representation of the structure of hadrons at the partonic level, correlating the longitudinal momentum fraction carried by the parton with its transverse spatial coordinate [5-9]. For recent theoretical reviews, see Refs. [10-13].

Generalized parton distributions depend on the squared four-momentum transfer $t$ to the nucleon and on $x$ and $\xi$, which represent, respectively, the average and half the difference of the longitudinal momentum fractions carried by the probed parton in initial and final states. Nucleon elastic form factors and parton distribution functions appear as $x$ moments and kinematic limits (for $t, \xi \rightarrow 0$ ) of GPDs, respectively. The skewness parameter $\xi$ is related to the Bjorken variable $x_{\mathrm{B}}=$ $Q^{2} /(2 M \nu)$, as $\xi \approx x_{\mathrm{B}} /\left(2-x_{\mathrm{B}}\right)$ in the Bjorken limit, where $Q^{2} \rightarrow \infty$ at fixed values of $x_{\mathrm{B}}$ and $t$. Here, $M$ is the target mass and $-Q^{2}$ is the squared four-momentum of the exchanged virtual photon with energy $v$ in the target rest frame. Most often discussed are the four twist-2 quark-helicity-conserving GPDs for each quark species in the nucleon: the quark-polarization averaged distributions $H$ and $E$ and the quark-polarization related distributions $\widetilde{H}$ and $\widetilde{E}$.

Among all presently practical hard exclusive probes, deeply virtual Compton scattering (DVCS), that is, the hard exclusive leptoproduction of a real photon, appears to have the most reliable interpretation in terms of GPDs. The final state of the DVCS process, in which the real photon is radiated by a quark, is intrinsically indistinguishable from

\footnotetext{
*Present address: Brookhaven National Laboratory, Upton, New York 11772-5000, USA.

${ }^{\dagger}$ Present address: Massachusetts Institute of Technology, Cambridge, Massachusetts 02139, USA.

${ }^{\ddagger}$ Present address: Institut für Physik, Universität Mainz, D-55128 Mainz, Germany.

§Present adress: Jefferson Lab, Newport News, Virginia 23606, USA.

"Present address: Institut für Kernphysik, Universität Frankfurt am Main, D-60438 Frankfurt am Main, Germany.

『klaus.rith@desy.de

*** Present address: Carnegie Mellon University, Pittsburgh, Pennsylvania 15213, USA.
}

PACS number(s): 13.60.Le, 13.85.Lg, 14.20.Dh

that of the Bethe-Heitler $(\mathrm{BH})$ process in which a real photon is radiated by the incoming or outgoing lepton. Access to the DVCS amplitude is provided by interference between the Bethe-Heitler and DVCS processes, for example, via the measurement of the cross-section asymmetries with respect to the lepton beam helicity and charge.

This paper reports the first experimental study of DVCS on nuclear targets. Nuclei provide a laboratory where, compared to the free nucleon, additional information on GPDs can be obtained by observing how they become modified in the nuclear environment. Therefore, studies of nuclear GPDs offer a new opportunity to investigate the nature of the nuclear environment.

In lepton-nucleus scattering, two processes can be distinguished for both DVCS and BH: (a) the coherent process, where the electron scatters off the whole nucleus, which stays intact; and (b) the incoherent process, where the electron scatters quasielastically from an individual nucleon, breaking up the nucleus.

For coherent scattering, various DVCS observables have been estimated theoretically [14,15]. In these estimates, nuclear GPDs are expressed in terms of nucleon GPDs convoluted with the distribution of nucleons in the nucleus. The $t$ dependence is modeled using nuclear elastic form factors. These models predict an enhancement of the beam-charge and beam-helicity asymmetries for spin- 0 and spin- $1 / 2$ nuclei compared to the case of a free proton.

Recently, coherent DVCS on nuclei has been suggested to provide new insights into the origin of the European Muon Collaboration (EMC) effect [16-18], as models that attempt to explain the EMC effect in the forward case $(t, \xi \rightarrow 0)$ also predict nuclear GPDs that differ from those of a free nucleon ("generalized" EMC effect). GPD models embodying PDFs that describe the EMC effect observed in inclusive DIS predict a much larger generalized EMC effect for DVCS observables [19-23]. In Ref. [22], this enhancement is attributed to the transverse motion of quarks in nuclear targets, while Ref. [23] relates the enhancement to mesonic degrees of freedom in hard reactions on nuclei, which have been invoked in the "pion excess" models to explain the EMC effect in inclusive DIS [16, $17,24]$. An observable found to be sensitive to mesonic degrees of freedom is the real part of the DVCS amplitude, which is predicted to depend strongly on the nuclear mass number $A$ [23].

Incoherent scattering is approximated by scattering on free nucleons. In the kinematic conditions of this experiment, scattering on the proton dominates, owing to the fact that the $\mathrm{BH}$ process dominates the single-photon production rate and the $\mathrm{BH}$ process on the neutron is suppressed because of the small electromagnetic form factors compared to those of the proton. Therefore the asymmetries for nuclei in the incoherent case are anticipated to be similar to those for the proton. The role of the neutron contribution was studied in Ref. [25]. It was shown to decrease the asymmetries measured in incoherent nuclear DVCS at larger values of $-t$. 


\section{FORMALISM}

The cross section for hard exclusive leptoproduction of real photons reads

$$
\frac{\mathrm{d} \sigma}{\mathrm{d} x_{\mathrm{B}} \mathrm{d} Q^{2} \mathrm{~d}|t| \mathrm{d} \phi}=\frac{x_{\mathrm{B}} e^{6}}{32(2 \pi)^{4} Q^{4}} \frac{|\mathrm{T}|^{2}}{\sqrt{1+\epsilon^{2}}},
$$

where $e$ represents the elementary charge, $\epsilon \equiv 2 x_{\mathrm{B}} M / Q$, and $\mathrm{T}$ is the total reaction amplitude. The azimuthal angle $\phi$ is defined as the angle between the lepton scattering plane and the photon production plane spanned by the trajectories of the virtual and real photons, following Ref. [26]. The scattering amplitudes of the DVCS and BH processes add coherently. The cross section is then proportional to the squared photonproduction amplitude, written as

$$
|\mathrm{T}|^{2}=\left|\mathrm{T}_{\mathrm{DVCS}}\right|^{2}+\left|\mathrm{T}_{\mathrm{BH}}\right|^{2}+\mathrm{I},
$$

where the interference term I is given by

$$
\mathrm{I}=\mathrm{T}_{\mathrm{DVCS}} \mathrm{T}_{\mathrm{BH}}^{*}+\mathrm{T}_{\mathrm{DVCS}}^{*} \mathrm{~T}_{\mathrm{BH}} \text {. }
$$

The $\mathrm{BH}$ amplitude $\mathrm{T}_{\mathrm{BH}}$ is calculable from measured elastic form factors of the (nucleon) nucleus when modeling the observables for the (in-)coherent process. At leading order in the fine structure constant $\alpha$ and for an unpolarized target, the squared $\mathrm{BH}$ amplitude $\left|\mathrm{T}_{\mathrm{BH}}\right|^{2}$ is independent of beam polarization and lepton charge. In contrast, the squared DVCS amplitude $\left|\mathrm{T}_{\mathrm{DVCS}}\right|^{2}$ and the interference term I depend on the beam helicity, while the interference term also depends on the lepton charge. For a longitudinally polarized lepton beam and unpolarized target, these dependences read [11]

$$
\begin{aligned}
\left|\mathrm{T}_{\mathrm{BH}}\right|^{2}= & \frac{K_{\mathrm{BH}}}{\mathcal{P}_{1}(\phi) \mathcal{P}_{2}(\phi)} \sum_{n=0}^{2}\left[c_{n}^{\mathrm{BH}} \cos (n \phi)\right] \\
\left|\mathrm{T}_{\mathrm{DVCS}}\right|^{2}= & \frac{1}{Q^{2}}\left(\sum_{n=0}^{2}\left[c_{n}^{\mathrm{DVCS}} \cos (n \phi)\right]+P_{\mathrm{b}} s_{1}^{\mathrm{DVCS}} \sin \phi\right) \\
\mathrm{I}= & \frac{-e_{\ell} K_{\mathrm{I}}}{\mathcal{P}_{1}(\phi) \mathcal{P}_{2}(\phi)}\left(\sum_{n=0}^{3}\left[c_{n}^{\mathrm{I}} \cos (n \phi)\right]\right. \\
& \left.+P_{\mathrm{b}}\left[s_{1}^{\mathrm{I}} \sin \phi+s_{2}^{\mathrm{I}} \sin (2 \phi)\right]\right) .
\end{aligned}
$$

Here, $P_{\mathrm{b}}$ denotes the longitudinal beam polarization, $e_{\ell}$ is the beam charge in units of the elementary charge, $\mathcal{P}_{1}(\phi)$ and $\mathcal{P}_{2}(\phi)$ are the known $\phi$-dependent lepton propagators in the $\mathrm{BH}$ process, and the kinematic factors read $K_{\mathrm{BH}}=$ $1 /\left[x_{\mathrm{B}}^{2} t\left(1+\epsilon^{2}\right)^{2}\right]$ and $K_{\mathrm{I}}=1 /\left(x_{\mathrm{B}} y t\right)$, with $y$ the fraction of the incident lepton energy carried by the virtual photon in the target rest frame. The dependences of the coefficients $c_{n}$ and $s_{n}$ on GPDs are given in Ref. [11] ${ }^{1}$ for a spin-1/2 target and in Ref. [27] for a spin-0 target. For a spin-1/2 target, and within the typical kinematic conditions of this experiment, the

\footnotetext{
${ }^{1}$ Note that the azimuthal angle $\phi$ defined here is different from the one used in Ref. [11] $\left(\phi=\pi-\phi_{[11]}\right)$.
}

coefficients related to only twist-2 quark GPDs appearing in the interference term can be approximated as

$$
\begin{aligned}
& c_{1}^{\mathrm{I}} \propto F_{1} \operatorname{Re} \mathcal{H}, \\
& c_{0}^{\mathrm{I}} \propto-\frac{\sqrt{-t}}{Q} c_{1}^{\mathrm{I}}, \\
& s_{1}^{\mathrm{I}} \propto F_{1} \operatorname{Im} \mathcal{H},
\end{aligned}
$$

where $\mathcal{H}$ denotes the Compton form factor, which is a convolution of the GPD $H$ with the hard scattering amplitude, and $F_{1}$ is the Dirac form factor.

\section{EXPERIMENT AND ANALYSIS}

In this paper we present a study of hard exclusive production of real photons in the reaction $e A \rightarrow e \gamma X$. Data were collected with the HERMES spectrometer [28] during the period 19972005. The $27.6 \mathrm{GeV}$ HERA electron or positron beam at DESY was scattered off gaseous hydrogen, helium, nitrogen, neon, krypton, and xenon targets (see Table I). (Results from a deuterium target will be reported elsewhere [29].) The HERA beam was transversely self-polarized owing to the Sokolov-Ternov mechanism [30]. Longitudinal polarization of the beam was obtained by using a pair of spin rotators located before and after the interaction region of HERMES. The beam helicity was reversed every few months. The beam polarization was measured by two independent HERA polarimeters [31,32] with a combined fractional systematic uncertainty of up to $3.4 \%$. This analysis makes use of the full data set with nuclear targets and a subset of data with a hydrogen target taken in the years 2000 and 2005, corresponding to approximately $130 \mathrm{pb}^{-1}\left(100 \mathrm{pb}^{-1}\right)$ for the positron (electron) sample. (The results from the full 1996-2005 hydrogen data set has been reported elsewhere [33].) For hydrogen, krypton, and xenon targets, data for both positron and electron beams are available.

A brief description of the event selection is given here. More details are given in Refs. [34] and [35]. Events were selected if exactly one photon and one charged track identified as the scattered lepton were detected. The hadron contamination in the lepton sample is kept below $1 \%$ by combining the information from a transition-radiation detector, a preshower scintillator detector, and an electromagnetic calorimeter. The kinematic

TABLE I. Targets used for this analysis, their spins, the corresponding integrated luminosity $L$, and the average polarization for the two helicity states of the beam. Note that the xenon target is composed mainly of the isotopes ${ }^{129} \mathrm{Xe}$ (spin-1/2), ${ }^{131} \mathrm{Xe}(\operatorname{spin}-3 / 2)$, and ${ }^{132,134} \mathrm{Xe}(\operatorname{spin}-0)$, with fractional contributions of $26 \%, 21 \%$, and $36 \%$, respectively. For all other targets, the admixture of isotopes with a spin different from that given here is less than $10 \%$.

\begin{tabular}{lcccc}
\hline \hline$A$ & Spin & $L\left(\mathrm{pb}^{-1}\right)$ & $\left\langle P_{b}\right\rangle \leftarrow$ & $\left\langle P_{b}\right\rangle \rightarrow$ \\
\hline $\mathrm{H}$ & $1 / 2$ & 227 & 0.50 & -0.51 \\
$\mathrm{He}$ & 0 & 32 & 0.56 & -0.52 \\
$\mathrm{~N}$ & 1 & 51 & 0.39 & -0.40 \\
$\mathrm{Ne}$ & 0 & 86 & 0.52 & -0.55 \\
$\mathrm{Kr}$ & 0 & 77 & 0.43 & -0.41 \\
$\mathrm{Xe}$ & $0,1 / 2,3 / 2$ & 47 & 0.32 & -0.38 \\
\hline \hline
\end{tabular}




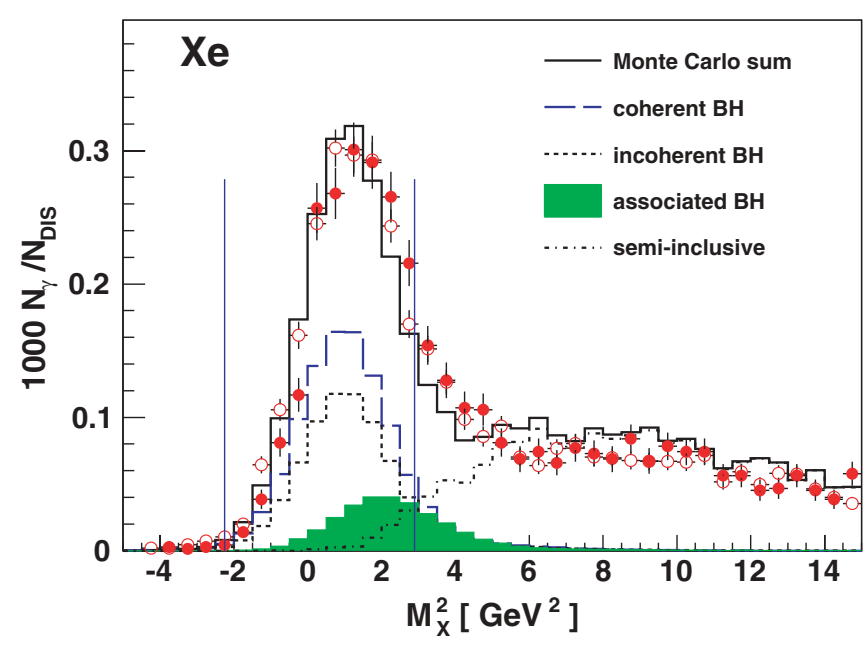

FIG. 1. (Color online) Distributions in squared missing mass from data using positron (filled circles) or electron (open circles) beams and a xenon target, compared to a MC simulation (solid line). The latter includes coherent Bethe-Heitler (BH) (dashed line), incoherent $\mathrm{BH}$ (short-dashed line), and associated $\mathrm{BH}$ [shaded (green) area] processes as well as the semi-inclusive background (dash-dotted line). The two solid vertical lines enclose the selected exclusive region for the positron data. See text for details.

requirements imposed are $1 \mathrm{GeV}^{2}<Q^{2}<10 \mathrm{GeV}^{2}, 0.03<$ $x_{\mathrm{B}}<0.35, v<22 \mathrm{GeV}$, and $W>3 \mathrm{GeV}$, where $W$ is the invariant mass of the virtual-photon/nucleon system. The real photon is identified by a "neutral cluster," which is defined as an energy deposition larger than $5 \mathrm{GeV}$ in the calorimeter and larger than $1 \mathrm{MeV}$ in the preshower detector and the absence of a corresponding charged track. The angular separation $\theta_{\gamma^{*} \gamma}$ between the virtual and the real photons is required to be larger than $2 \mathrm{mrad}$. This value is chosen to optimize the combined systematic and statistical uncertainties for the asymmetries owing to the degraded $\phi$ resolution at low $\theta_{\gamma^{*} \gamma}$ and the enhanced production of real photons on nuclear targets in the small $\theta_{\gamma^{*} \gamma}$ region $^{2}$ [36]. An upper bound of $45 \mathrm{mrad}$ is imposed on this angle to improve the signal-to-background ratio.

The recoiling system was not detected. Instead, an "exclusive" sample of events is selected by requiring the squared missing mass $M_{\mathrm{X}}^{2}=\left(q+p-q^{\prime}\right)^{2}$ to correspond within the experimental resolution to the squared proton mass. Here, $q\left(q^{\prime}\right)$ is the four-momentum of the virtual (real) photon and $p=\left(M_{\mathrm{p}}, \overrightarrow{0}\right)$, with $M_{\mathrm{p}}$ the proton mass. This selection criterion is chosen by means of a Monte Carlo (MC) simulation of the missing mass distribution. The result of the simulation is shown in comparison with the experimental data in Fig. 1. In the MC simulation the expressions in Eqs. 35 and 36 of Ref. [11] are used for the incoherent BH process. The simulation also takes into account the incoherent $\mathrm{BH}$ process, where a nucleon is excited to a resonant state (known as associated production) using a parametrization of the total $\gamma^{*} p$ cross section for the resonance region from Ref. [38] and calculating

\footnotetext{
${ }^{2}$ Note that this value is the only difference from earlier HERMES analyses, for which $\theta_{\gamma^{*} \gamma}>5 \mathrm{mrad}$.
}

the individual cross sections for single-meson decay channels, for example, $\Delta^{+} \rightarrow p \pi^{0}$, with the MAID2000 program [39]. For the coherent $\mathrm{BH}$ process, the parametrizations of the form factor for the respective nuclear targets are taken from Refs. [37] and [25]. In addition, semi-inclusive production of neutral mesons (mostly $\pi^{0}$ ) is included, where either only one photon from the $\pi^{0} \rightarrow \gamma \gamma$ decay is detected or these photons cannot be experimentally resolved. For this process, the MC generator LEPTO [40] is used in conjunction with a set of JETSET [41] fragmentation parameters that had previously been adjusted to reproduce multiplicity distributions observed by HERMES [42]. Not included in the simulation is radiation of more than one photon, which would move events from the peak to the continuum, nor the DVCS process. The latter contribution is highly model-dependent. In the GPD model used in Ref. [43] it varies between $10 \%$ and $25 \%$ of the BH yield for production from a hydrogen target [44].

The "exclusive region" for the positron data is defined as $-(1.5 \mathrm{GeV})^{2}<M_{\mathrm{X}}^{2}<(1.7 \mathrm{GeV})^{2}$, where the lower limit is chosen to be three times the resolution in $M_{\mathrm{X}}^{2}$ from the squared proton mass, and the upper limit to optimize the signal-tobackground ratio. Because the $M_{\mathrm{X}}^{2}$ spectrum of the electron data is found to be shifted by approximately $0.18 \mathrm{GeV}^{2}$ toward smaller values relative to that of the positron data, the exclusive region for electron data is shifted accordingly. One-quarter of the effect of this shift on the results presented subsequently is assigned as a contribution to the systematic uncertainty.

As the recoiling system was not detected, $t$ is inferred from the measurement of the other final-state particles. For elastic events, the kinematic relationship between the energy and the direction of the real photon permits the calculation of $t$ without using the measured energy of the real photon, which is the quantity subject to larger uncertainty. Thus the value of $t$ is calculated as

$$
t=\frac{-Q^{2}-2 v\left(v-\sqrt{v^{2}+Q^{2}} \cos \theta_{\gamma^{*} \gamma}\right)}{1+\frac{1}{M_{\mathrm{p}}}\left(v-\sqrt{v^{2}+Q^{2}} \cos \theta_{\gamma^{*} \gamma}\right)}
$$

for the exclusive event sample. The error caused by applying this expression to inelastic events is accounted for in the MC simulation that is used to calculate the fractional contribution of background processes per kinematic bin. The quantity $-t$ is required to be smaller than $0.7 \mathrm{GeV}^{2}$.

Coherent scattering on nuclear targets is separated from incoherent scattering by exploiting its characteristic $t$ dependence. For both DVCS and BH, coherent scattering occurs at small values of $-t$ and rapidly diminishes with increasing $|t|$. However, a complete separation of the two scattering processes is impossible at HERMES. Coherent-enriched and incoherentenriched samples are selected according to a $-t$ threshold that is chosen to vary with the target such that, for each sample, approximately the same average kinematic conditions are obtained for all targets. The kinematic distributions of elastic coherent and incoherent processes are determined using the MC simulation described previously and presented in Figs. 1 and 2 for xenon, as an example. The $t$ distribution of events selected in the exclusive region is shown in Fig. 2 for xenon together with the simulated contributions of coherent and incoherent processes. Tables II and III summarize the average 


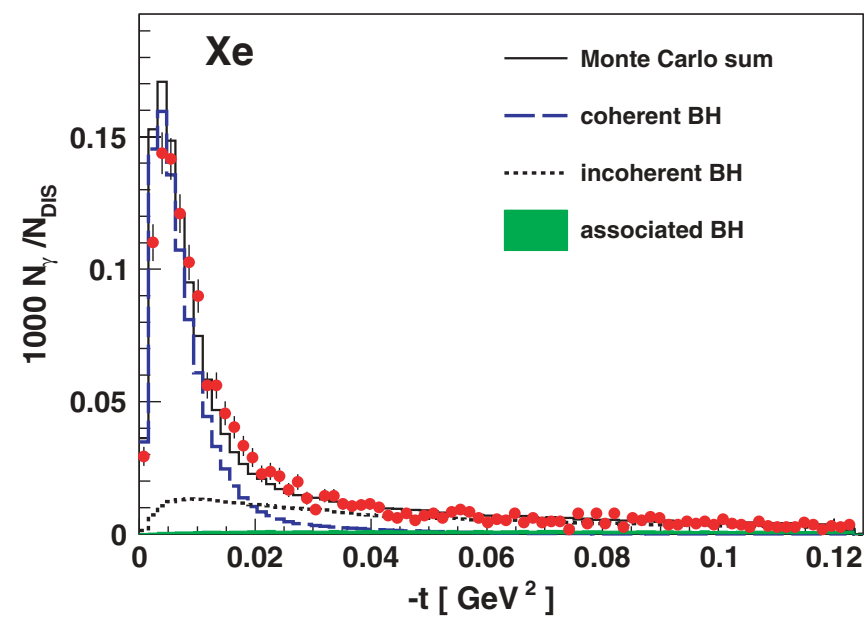

FIG. 2. (Color online) Distribution (points) of events selected in the exclusive region as a function of $-t$ compared to a MC simulation (solid line). The latter includes coherent Bethe-Heitler (BH) (dashed line), incoherent $\mathrm{BH}$ (dotted line), and associated $\mathrm{BH}$ [shaded (green) area] processes. Background from semi-inclusive neutral meson production is not included.

kinematic conditions for the various targets for the coherentenriched and incoherent-enriched samples, respectively, and list their purities defined as fractions of the total simulated yield. Also reported for each sample is the simulated fractional contribution from the associated $\mathrm{BH}$ process. For hydrogen, krypton, and xenon, the coherent-enriched region is further explored as a function of $t$ (see Table IV).

As coherent scattering could not be identified event by event for these data, kinematic variables that depend on the target mass are always calculated using the proton mass. This does not influence the selection of exclusive events, as the values of the relevant kinematic variables calculated using the proton mass are highly correlated with those calculated using the actual target mass. Also, the calculation of the $t$ value is affected negligibly.

The full cross section for exclusive production of real photons on unpolarized targets $(\mathrm{U})$ by a longitudinally polarized beam $(\mathrm{L})$ can be written as

$$
\begin{aligned}
\sigma(\phi)= & \sigma_{\mathrm{UU}, 0}(\phi)\left[1+e_{\ell} \mathcal{A}_{\mathrm{C}}(\phi)+P_{\mathrm{b}} \mathcal{A}_{\mathrm{LU}, \mathrm{DVCS}}(\phi)\right. \\
& \left.+e_{\ell} P_{\mathrm{b}} \mathcal{A}_{\mathrm{LU}, \mathrm{I}}(\phi)\right]
\end{aligned}
$$

TABLE II. Average kinematics and fractional contributions from coherent (coh.) processes (purity) and associated (assoc.) processes in the coherent-enriched sample for the various targets.

\begin{tabular}{lcccccc}
\hline \hline$A$ & $\begin{array}{c}t \text { threshold } \\
\left(\mathrm{GeV}^{2}\right)\end{array}$ & $\begin{array}{c}\langle t\rangle \\
\left(\mathrm{GeV}^{2}\right)\end{array}$ & $\left\langle x_{\mathrm{B}}\right\rangle$ & $\begin{array}{c}\left\langle Q^{2}\right\rangle \\
\left(\mathrm{GeV}^{2}\right)\end{array}$ & $\begin{array}{c}\% \text { of } \\
\text { coh. }\end{array}$ & $\begin{array}{c}\% \text { of } \\
\text { assoc. }\end{array}$ \\
\hline $\mathrm{H}$ & $-t<0.033$ & -0.018 & 0.070 & 1.81 & - & 4 \\
$\mathrm{He}$ & $-t<0.036$ & -0.018 & 0.072 & 1.83 & 34 & 4 \\
$\mathrm{~N}$ & $-t<0.043$ & -0.018 & 0.068 & 1.73 & 66 & 3 \\
$\mathrm{Ne}$ & $-t<0.044$ & -0.018 & 0.068 & 1.74 & 68 & 3 \\
$\mathrm{Kr}$ & $-t<0.070$ & -0.018 & 0.064 & 1.63 & 69 & 3 \\
$\mathrm{Xe}$ & $-t<0.078$ & -0.018 & 0.062 & 1.60 & 66 & 4 \\
\hline \hline
\end{tabular}

TABLE III. Average kinematics and fractional contributions from incoherent (incoh.) processes (purity) and associated (assoc.) processes in the incoherent-enriched sample for the various targets.

\begin{tabular}{lcccccc}
\hline \hline$A$ & $\begin{array}{c}t \text { threshold } \\
\left(\mathrm{GeV}^{2}\right)\end{array}$ & $\begin{array}{c}\langle t\rangle \\
\left(\mathrm{GeV}^{2}\right)\end{array}$ & $\left\langle x_{\mathrm{B}}\right\rangle$ & $\begin{array}{c}\left\langle Q^{2}\right\rangle \\
\left(\mathrm{GeV}^{2}\right)\end{array}$ & $\begin{array}{c}\% \text { of } \\
\text { incoh. }\end{array}$ & $\begin{array}{c}\% \text { of } \\
\text { assoc. }\end{array}$ \\
\hline $\mathrm{H}$ & $-t>0.077$ & -0.200 & 0.109 & 2.89 & - & 20 \\
$\mathrm{He}$ & $-t>0.084$ & -0.200 & 0.107 & 2.78 & 61 & 28 \\
$\mathrm{~N}$ & $-t>0.083$ & -0.200 & 0.113 & 2.93 & 60 & 28 \\
$\mathrm{Ne}$ & $-t>0.075$ & -0.200 & 0.111 & 2.92 & 65 & 28 \\
$\mathrm{Kr}$ & $-t>0.067$ & -0.200 & 0.108 & 2.84 & 57 & 30 \\
$\mathrm{Xe}$ & $-t>0.060$ & -0.200 & 0.107 & 2.86 & 56 & 30 \\
\hline \hline
\end{tabular}

where $\sigma_{\mathrm{UU}, 0}(\phi)=\frac{1}{4}\left[\sigma^{+\rightarrow}+\sigma^{-\rightarrow}+\sigma^{+\leftarrow}+\sigma^{-\leftarrow}\right]$ is the cross section for an unpolarized target averaged over both beam charges $(+,-)$ and over both positive $(\rightarrow)$ and negative $(\leftarrow)$ beam helicities. The beam-charge asymmetry $\mathcal{A}_{\mathrm{C}}$ and beam-helicity asymmetries $\mathcal{A}_{\mathrm{LU}, \mathrm{DVCS}}$ and $\mathcal{A}_{\mathrm{LU}, \mathrm{I}}$ are defined in Eqs. (12), (14), and (16), respectively. Each definition is complemented by the corresponding relation to the coefficients given in Eqs. (4)-(6):

$$
\begin{aligned}
\mathcal{A}_{\mathrm{C}}(\phi) & \equiv \frac{\sigma^{+\rightarrow}-\sigma^{-\rightarrow}+\sigma^{+\leftarrow}-\sigma^{-\leftarrow}}{\sigma^{+\rightarrow}+\sigma^{-\rightarrow}+\sigma^{+\leftarrow}+\sigma^{-\leftarrow}} \\
& =\frac{-1}{\sigma_{\mathrm{UU}, 0}(\phi)} \frac{K_{\mathrm{I}}}{\mathcal{P}_{1}(\phi) \mathcal{P}_{2}(\phi)} \sum_{n=0}^{3} c_{n}^{\mathrm{I}} \cos (n \phi), \\
\mathcal{A}_{\mathrm{LU}, \mathrm{DVCS}}(\phi) & \equiv \frac{\sigma^{+\rightarrow}+\sigma^{-\rightarrow}-\sigma^{+\leftarrow}-\sigma^{-\leftarrow}}{\sigma^{+\rightarrow}+\sigma^{-\rightarrow}+\sigma^{+\leftarrow}+\sigma^{-\leftarrow}} \\
& =\frac{1}{\sigma_{\mathrm{UU}, 0}(\phi)} \frac{1}{Q^{2}} s_{1}^{\mathrm{DVCS}} \sin \phi, \\
\mathcal{A}_{\mathrm{LU}, \mathrm{I}}(\phi) & \equiv \frac{\sigma^{+\rightarrow}-\sigma^{-\rightarrow}-\sigma^{+\leftarrow}+\sigma^{-\leftarrow}}{\sigma^{+\rightarrow}+\sigma^{-\rightarrow}+\sigma^{+\leftarrow}+\sigma^{-\leftarrow}} \\
& =\frac{-1}{\sigma_{\mathrm{UU}, 0}(\phi)} \frac{K_{\mathrm{I}}}{\mathcal{P}_{1}(\phi) \mathcal{P}_{2}(\phi)} \sum_{n=1}^{2} s_{n}^{\mathrm{I}} \sin (n \phi) .
\end{aligned}
$$

As the term in Eq. (11) including $\mathcal{A}_{\mathrm{LU}, \mathrm{I}}$ depends on both beam helicity and beam charge, the DVCS and interference beam-helicity asymmetries can be separated. Such a combined analysis [45] was performed for hydrogen, krypton, and xenon, where data for both electron and positron beams are available.

TABLE IV. Average kinematics and fractional contributions from coherent (coh.) processes (purity) and associated (assoc.) processes in two $t$ subranges of the coherent-enriched sample for hydrogen, krypton, and xenon.

\begin{tabular}{lcccccr}
\hline \hline$A$ & $\begin{array}{c}t \text { range } \\
\left(\mathrm{GeV}^{2}\right)\end{array}$ & $\begin{array}{c}\langle t\rangle \\
\left(\mathrm{GeV}^{2}\right)\end{array}$ & $\left\langle x_{\mathrm{B}}\right\rangle$ & $\begin{array}{c}\left\langle Q^{2}\right\rangle \\
\left(\mathrm{GeV}^{2}\right)\end{array}$ & $\begin{array}{c}\% \text { of } \\
\text { coh. }\end{array}$ & $\begin{array}{r}\% \text { of } \\
\text { assoc. }\end{array}$ \\
\hline $\mathrm{H}$ & $0.0<-t<0.008$ & -0.006 & 0.054 & 1.38 & - & $<1$ \\
$\mathrm{Kr}$ & $0.0<-t<0.010$ & -0.006 & 0.053 & 1.37 & 92 & $<1$ \\
$\mathrm{Xe}$ & $0.0<-t<0.010$ & -0.006 & 0.053 & 1.37 & 92 & $<1$ \\
$\mathrm{H}$ & $0.008<-t<0.020$ & -0.014 & 0.069 & 1.75 & - & 1 \\
$\mathrm{Kr}$ & $0.010<-t<0.020$ & -0.014 & 0.064 & 1.63 & 71 & 2 \\
$\mathrm{Xe}$ & $0.010<-t<0.020$ & -0.014 & 0.062 & 1.67 & 71 & 2 \\
\hline \hline
\end{tabular}


The asymmetries defined in Eqs. (12), (14), and (16) are expanded in terms of the following harmonics in $\phi$ :

$$
\begin{aligned}
\mathcal{A}_{\mathrm{C}}(\phi) \simeq & A_{\mathrm{C}}^{\cos (0 \phi)}+A_{\mathrm{C}}^{\cos \phi} \cos \phi+A_{\mathrm{C}}^{\cos (2 \phi)} \cos (2 \phi) \\
& +A_{\mathrm{C}}^{\cos (3 \phi)} \cos (3 \phi) \\
\mathcal{A}_{\mathrm{LU}, \mathrm{DVCS}}(\phi) \simeq & A_{\mathrm{LU}, \mathrm{DVCS}}^{\sin \phi} \sin \phi \\
\mathcal{A}_{\mathrm{LU}, \mathrm{I}}(\phi) \simeq & A_{\mathrm{LU}, \mathrm{I}}^{\sin \phi} \sin \phi+A_{\mathrm{LU}, \mathrm{I}}^{\sin (2 \phi)} \sin (2 \phi) .
\end{aligned}
$$

Using the method of maximum likelihood, the Fourier coefficients $A$, hereafter called asymmetry amplitudes, are simultaneously extracted from the event yield that is proportional to the cross section in Eq. (11). Although these asymmetry amplitudes differ somewhat from the coefficients in Eqs. (13), (15), and (17), they are well defined and can be computed in various GPD models for direct comparison with data.

For helium, nitrogen, and neon, only data with a positron beam were collected. The single-charge (positron) beamhelicity asymmetry is defined as

$$
\begin{aligned}
\mathcal{A}_{\mathrm{LU},+}(\phi) \equiv & \frac{\sigma^{\rightarrow}-\sigma^{\leftarrow}}{\sigma^{\rightarrow}+\sigma^{\leftarrow}} \\
= & \frac{1}{\sigma_{\mathrm{UU},+}(\phi)} \frac{1}{Q^{2}} s_{1}^{\mathrm{DVCS}} \sin \phi \\
& +\frac{-1}{\sigma_{\mathrm{UU},+}(\phi)} \frac{e_{\ell} K_{\mathrm{I}}}{\mathcal{P}_{1}(\phi) \mathcal{P}_{2}(\phi)} \sum_{n=1}^{2} s_{n}^{\mathrm{I}} \sin (n \phi),
\end{aligned}
$$

where $\sigma_{\mathrm{UU},+}(\phi)=\frac{1}{2}\left(\sigma^{\rightarrow}+\sigma^{\leftarrow}\right)$. In this case the event yield that is proportional to the cross section of Eq. (11) is fitted by

$$
\mathcal{A}_{\mathrm{LU},+} \simeq A_{\mathrm{LU},+}^{\sin \phi} \sin \phi+A_{\mathrm{LU},+}^{\sin (2 \phi)} \sin (2 \phi) .
$$

This method does not allow for a separation of squared DVCS amplitude and interference term in the beam-helicity asymmetry. It was used in an earlier extraction of beam-helicity asymmetries for hydrogen [46].

In each kinematic bin, the extracted asymmetry amplitudes are corrected for background from the decay of semi-inclusively produced neutral mesons, mainly pions. The corrected asymmetry amplitude is then obtained as

$$
A_{\text {corr }}=\frac{A_{\text {raw }}-f_{\text {semi }} \cdot A_{\text {semi }}}{1-f_{\text {semi }}},
$$

where $A_{\text {raw }}$ stands for the extracted raw asymmetry amplitude, and $f_{\text {semi }}$ and $A_{\text {semi }}$ for the fractional contribution and corresponding asymmetry amplitude of the semi-inclusive background, respectively. The background contribution $f_{\text {semi }}$, estimated from MC simulations, ranges from $1 \%$ to $11 \%$, depending on the kinematic conditions, and amounts to $3.5 \%$ on average. Because the beam-charge-dependent background asymmetry is zero at leading-order QED, the semi-inclusive background constitutes a dilution for $\mathcal{A}_{\mathrm{C}}$ and effectively also for $\mathcal{A}_{\mathrm{LU}, \mathrm{I}}$, as it cancels in the latter case. To correct $\mathcal{A}_{\mathrm{LU}, \mathrm{DVCS}}$ and $\mathcal{A}_{\mathrm{LU}}$ for the semi-inclusive background, the size of the corresponding beam-helicity asymmetry is extracted from data by reconstructing neutral pions with a large fractional energy $z=E_{\pi^{0}} / v>0.8$, as, according to MC simulations, only these contribute to the exclusive region [44]. These simulations show that the extracted $\pi^{0}$ asymmetry does not depend on whether only one or both photons are in the acceptance. One half of the size of the full background correction is assigned as systematic uncertainty. Contributions from exclusive $\pi^{0}$ production were found to be negligible at HERMES in an MC simulation based on a GPD model for exclusive meson production [47] as well as in a data search for exclusive $\pi^{0}$ production [48]. Hence this conceivable contribution is not included in the systematic uncertainty.

The asymmetry amplitude $A_{\text {corr }}$, determined by applying Eq. (24), is expected to originate from only elastic and associated production. Because essentially nothing is known about the asymmetry for associated production, no correction is made or uncertainty is assigned for the latter, but instead associated production is considered to be part of the signal in this analysis. The fractional contribution of associated processes is strongly $t$ dependent, ranging from 3\% in the lowest $t$ bin to $50 \%$ in the highest $t$ bin, with little dependence on $A$.

Effects from detector acceptance, kinematic smearing, finite bin width, and possible detector misalignment are estimated using a MC simulation based on the GPD model of Ref. [49]. Note that a mistake has been found in this GPD model [50]; however, the model describes well the magnitude and kinematic dependences of previously reported HERMES beam-charge [45] and beam-helicity asymmetries [33] and thus is considered to be suitable for systematic studies. For each bin in $-t$, the asymmetry amplitude for hydrogen is (i) calculated at the mean kinematic values of a given bin and (ii) extracted from the reconstructed MC simulation that includes all experimental effects. The difference between these two amplitude values is included in the systematic uncertainty. This uncertainty estimated for hydrogen is applied to all targets. The validity of this approach was checked using MC simulations based on the model in Ref. [49], which also parameterizes nuclear GPDs. The systematic uncertainty obtained for the nuclear targets is, within its statistical uncertainty, in good agreement with that estimated for hydrogen.

The total systematic uncertainty is dominated by the uncertainty from the combined contributions of detector acceptance, kinematic smearing, finite bin width, and possible detector misalignment. This combination is added in quadrature with contributions arising from the background correction and the relative energy shift between the $M_{\mathrm{X}}^{2}$ spectrum of positron and electron data. A scale uncertainty of up to $3.4 \%$ arising from beam polarimetry is not included in the systematic uncertainty for the beam helicity related asymmetries. Also not included is any contribution owing to additional QED vertices, as the most significant of these was estimated to be negligible in the case of helicity asymmetries [51].

\section{RESULTS AND DISCUSSION}

In Figs. 3-5, the amplitudes of the beam-charge and beamhelicity asymmetries, $A_{\mathrm{C}}^{\cos \phi}, A_{\mathrm{LU}, \mathrm{DVCS}}^{\sin \phi}, A_{\mathrm{LU}, \mathrm{I}}^{\sin \phi}$, and $A_{\mathrm{LU},+}^{\sin \phi}$, are 


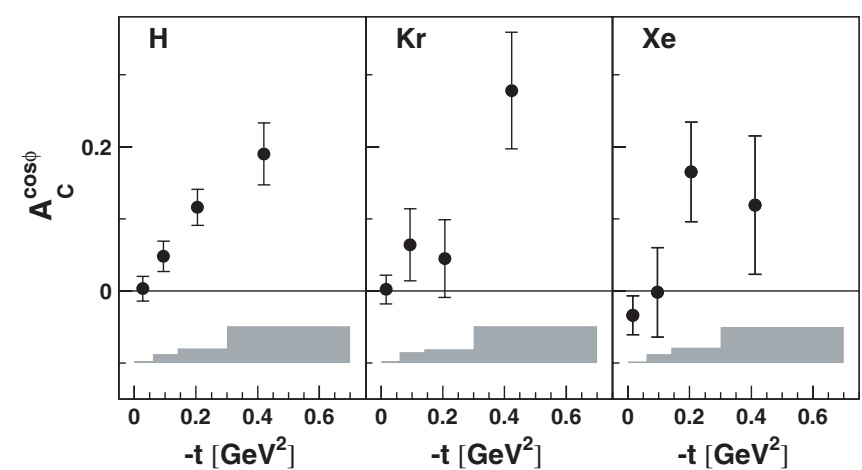

FIG. 3. The $\cos \phi$ amplitude of the beam-charge asymmetry for hydrogen, krypton, and xenon as a function of $t$. Error bars (bands) represent statistical (systematic) uncertainties.

shown as functions of $-t$ for unseparated coherent and incoherent production. For the nuclear targets, all other amplitudes in Eqs. (18)-(20) and (23) are found to be consistent with zero within $1.5 \sigma$ of the statistical uncertainty. These other asymmetry amplitudes relate to coefficients that either embody higher-twist quark GPDs or are kinematically suppressed, as, for example, the amplitude presented in Eq. (8).

Figure 3 shows the amplitude $A_{\mathrm{C}}^{\cos \phi}$ for hydrogen, krypton, and xenon. The values for hydrogen from this analysis are consistent with those extracted previously [35,45]. For hydrogen, krypton, and xenon, the availability of data with both beam charges allows for separation of the azimuthal harmonics of the squared DVCS amplitude and the interference term. The beamhelicity amplitude $A_{\mathrm{LU}, \mathrm{DVCS}}^{\sin }$, shown in Fig. 4, is consistent with zero for all three targets over the full $-t$ range. This is in agreement with the expected suppression of the amplitude.

The beam-helicity amplitudes $A_{\mathrm{LU}, \mathrm{I}}^{\sin \phi}$ and $A_{\mathrm{LU},+}^{\sin }$, shown in Fig. 5, are substantial for all targets. For helium, nitrogen, and neon, where only positron beam data are available, this amplitude also receives contributions from the squared-DVCS term. However, as the latter amplitude is expected to be suppressed, and found to be so for other targets in Fig. 4, its contribution is assumed to be small here.

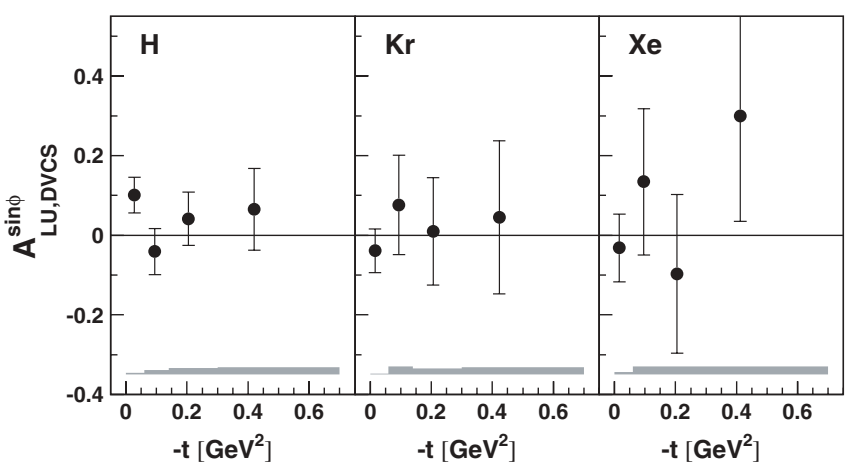

FIG. 4. The $\sin \phi$ amplitude of the beam-helicity asymmetry sensitive to the squared DVCS amplitude for hydrogen, krypton, and xenon as function of $t$. Error bars (bands) represent statistical (systematic) uncertainties. This amplitude is subject to an additional $3.4 \%$ maximal scale uncertainty arising from beam polarimetry.

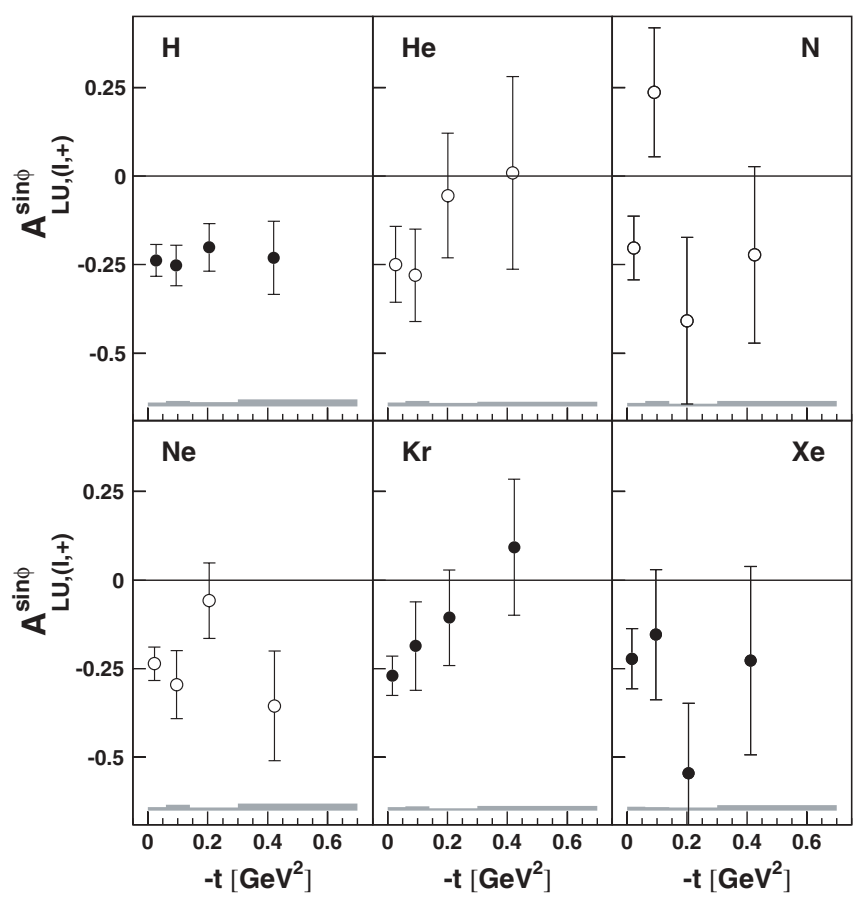

FIG. 5. The $t$ dependence of the $\sin \phi$ amplitude of the beamhelicity asymmetry sensitive to the interference term, $A_{\mathrm{LU}, \mathrm{I}}^{\sin \phi}$, for hydrogen, krypton, and xenon (filled symbols) or to a linear combination of the interference and the squared DVCS amplitude, $A_{\mathrm{LU},+}^{\sin \phi}$, for helium, nitrogen, and neon (open symbols). Error bars (bands) represent statistical (systematic) uncertainties. This amplitude is subject to an additional $3.4 \%$ maximal scale uncertainty arising from beam polarimetry.

The nuclear-mass dependence of the azimuthal beamcharge and beam-helicity asymmetries is presented separately for the coherent- and incoherent-enriched samples in Figs. 6 and 7. The $\cos \phi$ amplitude of the beam-charge asymmetry is consistent with zero for the coherent-enriched samples for all three targets, while it is about 0.1 for the incoherent-enriched samples, without showing any dependence on the target mass within uncertainties. The $\sin \phi$ amplitude of the beam-helicity asymmetry shown in Fig. 7 has values of about -0.2 for both the coherent- and the incoherent-enriched samples, without showing any dependence on $A$ within uncertainties. To quantify nuclear effects, the asymmetry amplitudes for nuclear targets are compared to those for a free proton. The ratio $R_{\mathrm{LU}}=A_{\mathrm{LU},(\mathrm{I},+), \mathrm{A}}^{\sin \phi} / A_{\mathrm{LU}, \mathrm{I}, \mathrm{H}}^{\sin \phi}$ of the nuclear-to-hydrogen beamhelicity asymmetry amplitudes, averaged over all targets, is found to be $0.91 \pm 0.19$ for the coherent-enriched sample and $0.93 \pm 0.23$ for the incoherent-enriched sample, both of which are compatible with unity.

For incoherent scattering, the asymmetry for nuclei is expected to be similar to that for hydrogen aside from effects of the nuclear environment, as scattering on a proton dominates. Neglecting the neutron contribution, the value of $R_{\mathrm{LU}}$ for incoherent scattering is expected to be unity [15]. In Ref. [25], the neutron contribution to incoherent nuclear DVCS is taken into account and $R_{\mathrm{LU}}$ is predicted to be between 0.66 and 0.74 at $t=-0.2 \mathrm{GeV}^{2}$. Within the experimental uncertainties, the measured ratio $R_{\mathrm{LU}}=0.93 \pm 0.23$ agrees both with the 


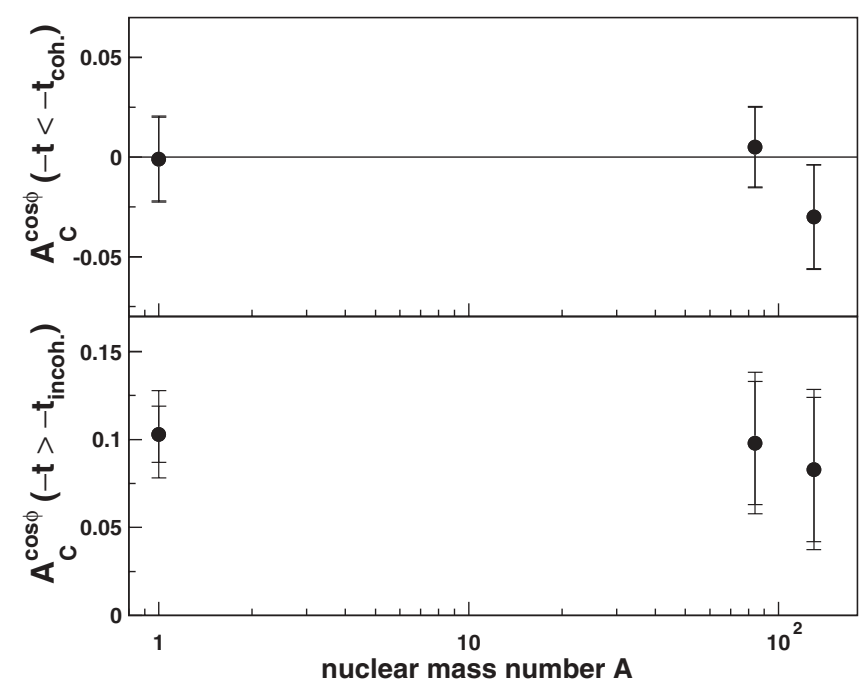

FIG. 6. Nuclear-mass dependence of the $\cos \phi$ amplitude of the beam-charge asymmetry for coherent-enriched (upper panel) and incoherent-enriched (lower panel) data samples for hydrogen, krypton, and xenon. Coherent-enriched samples have a purity of about $67 \%$; incoherent-enriched samples, a purity of about $60 \%$. Inner error bars represent the statistical uncertainty; full bars, the quadratic sum of statistical and systematic uncertainties.

expected suppression of the neutron contribution in incoherent scattering on nuclei and with the prediction of Ref. [25].

The results for the coherent-enriched samples can be compared to the predictions based on simple models for nuclear GPDs that express them in terms of nucleon GPDs [14,15]. Within this approach, nuclear beam-charge and beam-helicity asymmetries are predicted to be essentially independent of $A$ for heavier nuclei. Compared to the free proton asymmetry, the nuclear beam-charge and beam-helicity asymmetries are expected to be enhanced for spin- 0 and spin- $1 / 2$ nuclei. This predicted enhancement is based on the model-independent observation that DVCS takes place either on a proton or on a neutron in the nuclear target, while $\mathrm{BH}$ occurs predominantly only on a proton. The ratio $R_{\mathrm{LU}}$ of the nuclear-to-hydrogen beam-helicity asymmetry amplitudes has been estimated in Ref. [14] for the pure coherent process to be about $5 / 3$ for spin0 and spin- $1 / 2$ nuclei with $Z=N$, essentially independent of $A$. This value arises from the ratio of squared charges for an isoscalar to an isodoublet state and the observation that for the valence quark PDFs $d / u=1 / 2$ in the kinematics of this experiment. For spin-1 nuclei, $R_{\mathrm{LU}}$ is predicted to be unity. Ref. [14] also formulates a GPD model. Considering only leading twist GPDs and valence quark contributions, the predicted value of $A_{\mathrm{LU}, \mathrm{I}}^{\sin \phi}$ for hydrogen is -0.26 for the kinematic condition $t=-0.2 \mathrm{GeV}^{2}, Q^{2}=2.5 \mathrm{GeV}^{2}$, and $x_{\mathrm{B}}=0.12$. Including sea quark contributions and twist-3 corrections and varying the main model parameters, the predicted amplitude is in the range $0.16 \leqslant\left|A_{\mathrm{LU}, \mathrm{I}}^{\sin \phi}\right| \leqslant 0.37$. In Ref. [15], a somewhat more elaborated calculation is presented, where nuclear GPDs are expressed in terms of nucleon GPDs convoluted with the distribution of nucleons in the nucleus, thereby accounting for nuclear binding. Within this approach, the ratio $R_{\mathrm{LU}}$ is predicted to be about 1.8 for neon and krypton for the

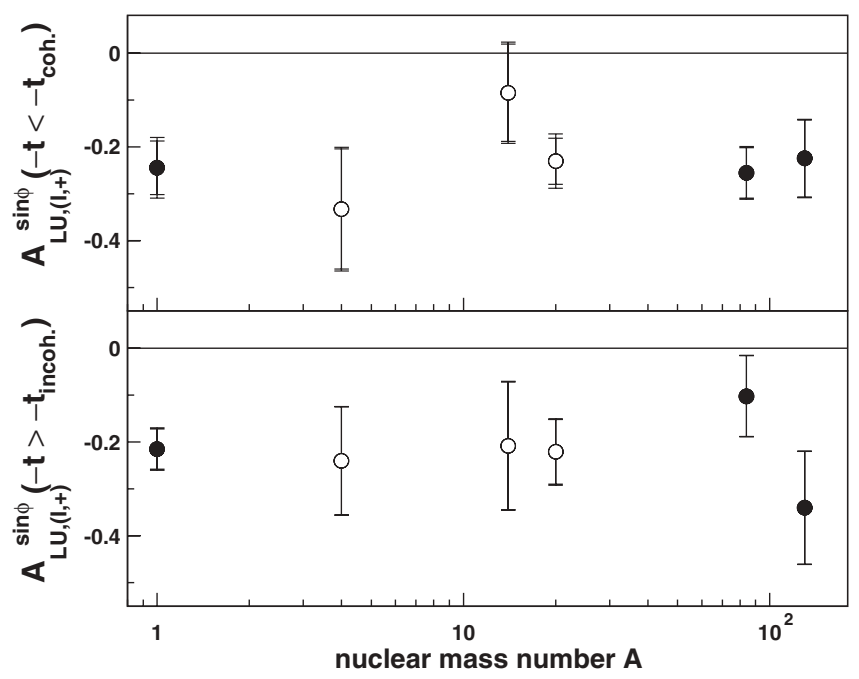

FIG. 7. Nuclear-mass dependence of the $\sin \phi$ amplitude of the beam-helicity asymmetry for coherent-enriched (upper panel) and incoherent-enriched (lower panel) data samples. See Fig. 5 caption for the meaning of open and filled circles. Coherent-enriched samples have a purity of about $67 \%$ except for $\mathrm{He}$, at $34 \%$; incoherent-enriched samples, a purity of about $60 \%$. Inner error bars represent the statistical uncertainty; full bars, the quadratic sum of statistical and systematic uncertainties. This amplitude is subject to an additional $3.4 \%$ maximal scale uncertainty arising from beam polarimetry.

kinematic condition $t=-0.018 \mathrm{GeV}^{2}, Q^{2}=1.58 \mathrm{GeV}^{2}$, and $x_{\mathrm{B}}=0.10$.

The nuclear beam-helicity amplitudes shown in Fig. 7 (upper panel) support the predicted independence of $A$ for heavier targets. They do not support the anticipated enhancement of the asymmetries compared to the free proton asymmetries for spin- 0 and spin- $1 / 2$ nuclei. However, the measured amplitude for the coherent-enriched sample receives contributions from incoherent scattering, which is expected to diminish $R_{\mathrm{LU}}$. The value $R_{\mathrm{LU}}=0.91 \pm 0.19$ for the coherent-enriched samples should therefore be compared to a prediction involving a mixture of asymmetry amplitudes for coherent and incoherent processes. For an average purity of $67 \%$ for the coherentenriched samples of nitrogen to xenon (see Table II) and assuming that the asymmetry from the incoherent portion of

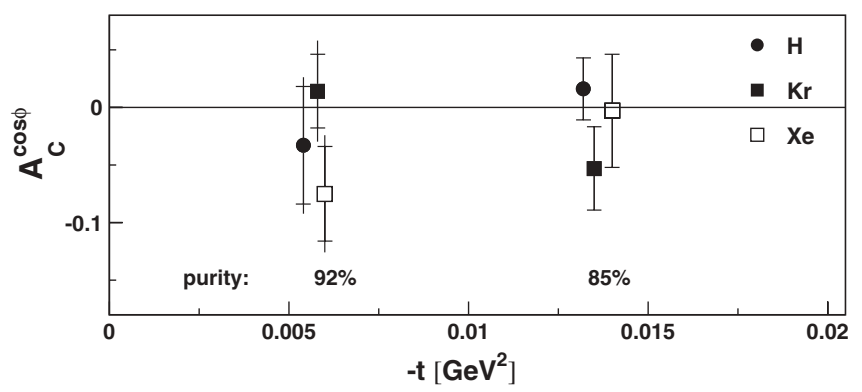

FIG. 8. The $\cos \phi$ amplitude of the beam-charge asymmetry for hydrogen, krypton, and xenon as a function of $t$. Inner error bars represent the statistical uncertainty; full bars, the quadratic sum of statistical and systematic uncertainties. The purity of the coherentenriched $\mathrm{Kr}$ and $\mathrm{Xe}$ samples is indicated for the two $t$ bins. 


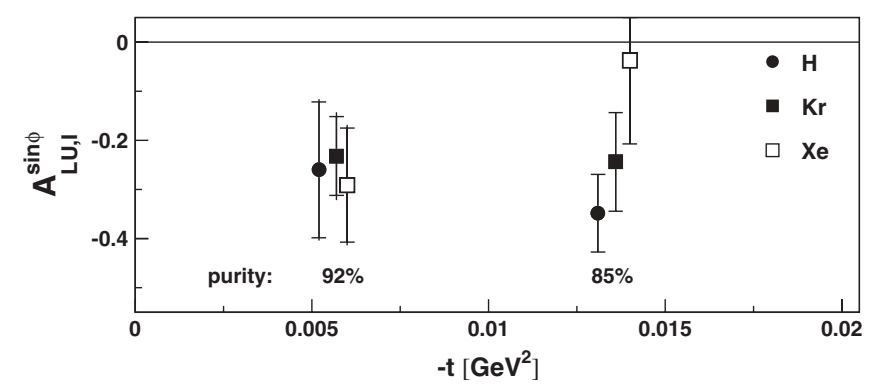

FIG. 9. The $\sin \phi$ amplitude of the beam-helicity asymmetry sensitive to the interference term for hydrogen, krypton, and xenon as a function of $t$. This amplitude is subject to an additional $3.4 \%$ maximal scale uncertainty arising from beam polarimetry. Otherwise, as Fig. 8.

the yield is the same as for hydrogen, the predicted ratio $R_{\mathrm{LU}}=$ $5 / 3$ for the pure coherent process becomes 1.45. In Ref. [15], both coherent and incoherent scattering are considered and the predicted ratio $R_{\mathrm{LU}}$ of about 1.8 for the pure coherent process becomes about 1.6. These values exceed the measured ratio by more than three standard deviations of the total experimental uncertainty. As shown in Figs. 8 and 9, for coherent-enriched samples both beam-charge and beam-helicity amplitudes for hydrogen, krypton, and xenon are essentially independent of $t$ within uncertainties.

In Ref. [23], mesonic degrees of freedom are also considered in the description of coherent scattering on nuclei and in the explanation of the generalized EMC effect. Such a contribution is predicted to significantly enhance the real part of the DVCS amplitude, which translates into a strong nuclear-mass dependence of the beam-charge asymmetry. In the absence of meson exchange, this asymmetry is expected to be essentially independent of $A$ for heavier nuclei. The nuclear beam-charge amplitudes shown in Fig. 6 (upper panel) do not show any enhancement about the amplitude for the free proton and do not exhibit any dependence on $A$.

\section{SUMMARY}

The nuclear-mass dependence of azimuthal beam-helicity asymmetries in electroproduction of real photons has been measured for the first time for targets ranging from hydrogen to xenon. For hydrogen, krypton, and xenon, data were taken with both beam charges and beam helicities allowing a separation of the $\sin \phi$ amplitude of the squared DVCS and the interference terms. Also, the $\cos \phi$ amplitude of the beam-charge asymmetry has been evaluated for those targets. This amplitude is consistent with earlier measurements for hydrogen $[35,45]$.
For the coherent-enriched data sample, the $\cos \phi$ amplitude is found to be consistent with zero for all nuclear targets, while it amounts to 0.1 for the incoherent-enriched data sample, in either case not exhibiting any dependence on $A$ within experimental uncertainties.

The $\sin \phi$ amplitude of the beam-helicity asymmetry sensitive to the squared DVCS amplitude is consistent with zero for all targets. The $\sin \phi$ amplitude of the beam-helicity asymmetry sensitive to the interference term is significantly nonzero, with a value of about -0.2 for both the coherentand the incoherent-enriched samples, without showing any dependence on $A$ within uncertainties. These amplitudes are compared to those of a free proton. The ratio $R_{\mathrm{LU}}=$ $A_{\mathrm{LU},(\mathrm{I},+), \mathrm{A}}^{\sin } / A_{\mathrm{LU}, \mathrm{I}, \mathrm{H}}^{\sin \phi}$ is found to be $0.91 \pm 0.19$ for the coherentenriched sample and $0.93 \pm 0.23$ for the incoherent-enriched sample, both of which are compatible with unity.

No nuclear-mass dependence of the beam-charge and beam-helicity asymmetries is observed for heavier nuclei, in agreement with the general feature of models that approximate nuclear GPDs by a sum of nucleon GPDs convoluted with the distribution of nucleons in the nucleus. The data do not support the enhancement of nuclear asymmetries compared to the free proton asymmetries for coherent scattering on spin- 0 and spin-1/2 nuclei as anticipated by various models [14,15,23].

\section{ACKNOWLEDGMENTS}

We thank M. Diehl, S. Liuti, and M. Siddikov for many interesting discussions. We gratefully acknowledge the DESY management for its support and the staff at DESY and the collaborating institutions for their significant effort. This work was supported by the FWO-Flanders and IWT, Belgium; the Natural Sciences and Engineering Research Council of Canada; the National Natural Science Foundation of China; the Alexander von Humboldt Stiftung; the German Bundesministerium für Bildung und Forschung (BMBF); the Deutsche Forschungsgemeinschaft (DFG); the Italian Istituto Nazionale di Fisica Nucleare (INFN); the MEXT, JSPS, and G-COE of Japan; the Dutch Foundation for Fundamenteel Onderzoek der Materie (FOM); the UK Engineering and Physical Sciences Research Council, the Science and Technology Facilities Council, and the Scottish Universities Physics Alliance; the US Department of Energy (DOE) and the National Science Foundation (NSF); the Russian Academy of Science and the Russian Federal Agency for Science and Innovations; the Ministry of Economy and the Ministry of Education and Science of Armenia; and the European Community-Research Infrastructure Activity under the FP6 "Structuring the European Research Area" program (HadronPhysics, Contract No. RII3-CT-2004-506078).
[1] D. Müller et al., Fortschr. Phys. 42, 101 (1994).

[2] A. V. Radyushkin, Phys. Lett. B380, 417 (1996).

[3] X. Ji, Phys. Rev. Lett. 78, 610 (1997).

[4] M. Diehl, T. Gousset, B. Pire, and J. P. Ralston, Phys. Lett. B411, 193 (1997).

[5] M. Burkardt, Phys. Rev. D 62, 071503(R) (2000); 66, 119903(E) (2002).
[6] M. Diehl, Eur. Phys. J. C 25, 223 (2002); 31, 277(E) (2003).

[7] J. P. Ralston and B. Pire, Phys. Rev. D 66, 111501(R) (2002).

[8] A. V. Belitsky and D. Müller, Nucl. Phys. A711, 118 (2002).

[9] M. Burkardt, Int. J. Mod. Phys. A 18, 173 (2003).

[10] K. Goeke, M. V. Polyakov, and M. Vanderhaeghen, Prog. Part. Nucl. Phys. 47, 401 (2001). 
[11] A. V. Belitsky, D. Müller, and A. Kirchner, Nucl. Phys. B629, 323 (2002).

[12] M. Diehl, Phys. Rep. 388, 41 (2003).

[13] A. V. Belitsky and A. V. Radyushkin, Phys. Rep. 418, 1 (2005).

[14] A. Kirchner and D. Müller, Eur. Phys. J. C 32, 347 (2003).

[15] V. Guzey and M. I. Strikman, Phys. Rev. C 68, 015204 (2003).

[16] L. L. Frankfurt and M. I. Strikman, Phys. Rep. 160, 235 (1988).

[17] D. F. Geesaman, K. Saito, and A. W. Thomas, Annu. Rev. Nucl. Part. Sci. 45, 337 (1995).

[18] P. R. Norton, Rep. Prog. Phys. 66, 1253 (2008).

[19] E. R. Berger, F. Cano, M. Diehl, and B. Pire, Phys. Rev. Lett. 87, 142302 (2001).

[20] F. Cano and B. Pire, Eur. Phys. J. A 19, 423 (2004).

[21] S. Scopetta, Phys. Rev. C 70, 015205 (2004); Nucl. Phys. A55, 523 (2005); Phys. Rev. C 79, 025207 (2009).

[22] S. Liuti and S. K. Taneja, Phys. Rev. C 72, 032201(C) (2005); 72, 034902 (2005).

[23] V. Guzey and M. Siddikov, J. Phys. G 32, 251 (2006).

[24] M. Arneodo, Phys. Rep. 240, 301 (1994).

[25] V. Guzey, Phys. Rev. C 78, 025211 (2008).

[26] A. Bacchetta, U. D’Alesio, M. Diehl, and C. A. Miller, Phys. Rev. D 70, 117504 (2004).

[27] A. V. Belitsky, D. Müller, A. Kirchner, and A. Schäfer, Phys. Rev. D 64, 116002 (2001).

[28] K. Ackerstaff et al. (HERMES Collaboration), Nucl. Instrum. Methods A 417, 230 (1998).

[29] A. Airapetian et al. (HERMES Collaboration), Nucl. Phys. B829, 1 (2010).

[30] A. A. Sokolov and I. M. Ternov, Sov. Phys. Dokl. 8, 1203 (1964).

[31] D. Barber et al., Nucl. Instrum. Methods A 338, 166 (1994).
[32] M. Beckmann et al., Nucl. Instrum. Methods A 479, 334 (2002).

[33] A. Airapetian et al. (HERMES Collaboration), JHEP 11 (2009) 083.

[34] F. Ellinghaus, Ph.D. thesis, Humboldt University Berlin, 2004 (DESY-THESIS-2004-005).

[35] A. Airapetian et al. (HERMES Collaboration), Phys. Rev. D 75, 011103(R) (2007).

[36] H. Ye, Ph.D. thesis, Peking University, 2008.

[37] R. F. Frosch et al., Phys. Rev. 160, 874 (1967).

[38] F. W. Brasse et al., Nucl. Phys. B110, 413 (1976).

[39] D. Drechsel, O. Hanstein, S. S. Kamalov, and L. Tiator, Comput. Phys. Commun. 101, 108 (1997).

[40] G. Ingelman, A. Edin, and J. Rathsman, Comput. Phys. Commun. 101, 108 (1997).

[41] T. Sjöstrand, Comput. Phys. Commun. 82, 74 (1994).

[42] A. Hillenbrand and M. Hartig, for the HERMES Collaboration, Proceedings of 40th Rencontres de Moriond on QCD and High Energy Hadronic Interactions, La Thuile, Aosta Valley, Italy, 12-19 March 2005; hep-ex/0505086.

[43] V. A. Korotkov and W.-D. Nowak, Eur. Phys. J. C 23, 455 (2002).

[44] Z. Ye, Ph.D. thesis, University Hamburg, 2006 (DESY-THESIS2007-005).

[45] A. Airapetian et al. (HERMES Collaboration), JHEP 06 (2008) 066.

[46] A. Airapetian et al. (HERMES Collaboration), Phys. Rev. Lett. 87, 182001 (2001).

[47] M. Vanderhaegen, P. A. M Guichon, and M. Guidal, Phys. Rev. D 60, 094017 (1999).

[48] A. Vandenbroucke, Ph.D. thesis, University of Gent, 2006 (DESY-THESIS-2007-003)

[49] V. Guzey and T. Teckentrup, Phys. Rev. D 74, 054027 (2006).

[50] V. Guzey and T. Teckentrup, Phys. Rev. D 79, 017501 (2009).

[51] A. V. Afanasev, M. I. Konchatnij, and N. P. Merenkov, J. Exp. Theor. Phys. 102, 220 (2006). 